\title{
The Prognostic Role of the Neutrophil to Lymphocyte Ratio at Recurrence in Esophageal Squamous Cell Carcinoma: Challenges and Future Directions
}

\author{
Audrey E. Kam, MD, and Ashiq Masood, MD \\ Division of Hematology, Oncology and Cell Therapy, Rush University Medical Center, Chicago, IL
}

Esophageal cancer is the eighth most commonly diagnosed malignancy worldwide and is the sixth leading cause of cancer-related mortality. ${ }^{1}$ Globally, esophageal squamous cell carcinoma (ESCC) is the most common histologic subtype and has been linked primarily to tobacco use, alcohol intake, and malnutrition. ${ }^{2}$ ESCC is most prevalent in East and Central Asia, where it comprises greater than $90 \%$ of esophageal cancers. ${ }^{1,2}$ Despite advances in multimodality treatment over the years, the prognosis of ESCC remains poor with a 5-year overall survival rate of $15-25 \% .^{3,4}$

The prognosis of ESCC patients has been tied to a variety of inflammatory biomarkers across several studies. $^{3-13}$ The systemic inflammatory response plays an integral role in DNA damage, cell proliferation, angiogenesis, and metastasis. ${ }^{3-9}$ Inflammatory biomarkers, such as neutrophil to lymphocyte ratio (NLR), lymphocyte to monocyte ratio (LMR), and platelet to lymphocyte ratio (PLR), have shown to predict survival in ESCC in multiple retrospective studies. ${ }^{3}-9$ The change in NLR (delta NLR) from time of diagnosis also has shown to predict response to neoadjuvant therapy, time to recurrence, and survival. ${ }^{3,9}$ Several meta-analyses also have confirmed the role of NLR as a prognostic biomarker in ESCC..$^{10-12}$ In addition to the NLR, the modified Glasgow Prognostic Score (mGPS),

(C) Society of Surgical Oncology 2021

First Received: 11 January 2021

Accepted: 14 January 2021;

Published Online: 9 February 2021

A. E. Kam, MD

e-mail: Audrey_E_Kam@rush.edu which combines albumin and C-reactive protein (CRP), has been demonstrated to predict survival in retrospective studies across multiple cancers, including ESCC.

In the current study by Shota et al. ${ }^{14}$ authors conducted a single institution, retrospective study of Japanese patients between 2004 and 2019 to evaluate the prognostic role of NLR at the time of recurrence in ESCC patients. A total of 133 patients were included in the analysis. On the multivariate analysis (MVA), the NLR at recurrence were significantly associated with worse overall survival (hazard ratio [HR] 1.061, 95\% confidence interval [CI] $1.002-1.125 ; p=0.043)$, whereas the mGPS was not. The optimal cutoff value for NLR at recurrence was set at 3.374 and was determined by survival classification and regression tree (CART) analysis. Time-dependent ROC curves demonstrated that the area under the curve (AUC) value of the NLR at recurrence was superior to mGPS at recurrence in all terms.

This publication is unique in that the majority of the aforementioned studies reported on the preoperative and postoperative NLR in ESCC, but not the NLR at time of recurrence. In addition, this variable may be complimentary to other parameters used to assess prognosis, such as time to recurrence, number of lymph nodes, and sites of recurrence.

While the results of this study are interesting, some limitations are noteworthy. First, the study was a singleinstitution, retrospective study restricted to Japanese patients, which lends itself to selection bias and makes it difficult to generalize to other patient populations. Second, the NLR may be affected by several other clinical variables that were not accounted for in the study, including immunosuppression, inflammation, infection, medications (e.g., steroids), and medical conditions (e.g., diabetes, thyroid dysfunction, hypertension, renal/liver failure). ${ }^{15}$ 
Third, the MVA did not include important parameters, such as performance status, smoking status, and medical comorbidities. Furthermore, serial longitudinal evaluation of the NLR over time may be more informative compared with the NLR at recurrence. Independent of this study, the wide applicability of NLR also is challenged by the large variation in cutoff values seen in the literature ranging between 1.7 and $5 .^{12}$

It is important to recognize the evolving role of precision oncology in clinical practice. With the advent of nextgeneration sequencing (NGS), serial longitudinal assessment of circulating tumor DNA (ctDNA) has been used to monitor response to therapy and to detect early recurrence. Studies have demonstrated that ESCC patients with posttreatment positive-ctDNA have a higher risk of recurrence, tumor progression, decreased progression-free survival, and lower overall survival. ${ }^{16,17}$ In addition, detection of ctDNA during surveillance has shown to precede radiographic evidence of recurrence by approximately 3-6 months. ${ }^{16,18}$ Thus, ctDNA analysis has the potential to serve as a useful prognostic tool in ESCC, albeit, larger prospective studies are needed to confirm this.

In conclusion, Shota et al. demonstrate that the NLR at time of recurrence may serve as a prognostic biomarker in ESCC in a Japanese patient cohort. ${ }^{14}$ However, study findings are limited by the retrospective nature of the study, the small and select patient population, the lack of inclusion of other clinical variables in the analysis, and the wide variability in NLR cutoff values seen across studies. Therefore, while the NLR at time of recurrence may play a prognostic role in ESCC, larger, prospective, multi-institutional studies will need to clarify its role in treatment decisions. Lastly, ctDNA analysis may represent a more powerful tool for predicting recurrence and survival in ESCC.

DISCLOSURES The authors disclose no conflicts of interest.

\section{REFERENCES}

1. Napier KJ, Scheerer M, Misra S. Esophageal cancer: a review of epidemiology, pathogenesis, staging workup and treatment modalities. World J Gastrointest Oncol. 2014;6(5):112-20.

2. The global, regional, and national burden of oesophageal cancer and its attributable risk factors in 195 countries and territories, 1990-2017: a systematic analysis for the Global Burden of Disease Study 2017.

3. Ishibashi Y, Tsujimoto H, Hiraki S, et al. Predictive value of immuno-inflammatory and nutritional measures modulated by neoadjuvant chemotherapy on the response of neoadjuvant chemotherapy and long-term outcomes in patients with esophageal cancer. Oncol Lett. 2020;19:487-97.
4. Duan H, Zhang X, Wang FX, et al. Prognostic role of neutrophillymphocyte ratio in operable esophageal squamous cell carcinoma. World J Gastroenterol. 2015;21(18):5591-7.

5. Ji WH, Jiang YH, Ji YL, et al. Prechemotherapy neutrophil:lymphocyte ratio is superior to the platelet:lymphocyte ratio as a prognostic indicator for locally advanced esophageal squamous cell cancer treated with neoadjuvant chemotherapy. Dis Esophagus. 2016;29(5):403-11.

6. Zhou XL, Li YQ, Zhu WG, et al. Neutrophil-to-lymphocyte ratio as a prognostic biomarker for patients with locally advanced esophageal squamous cell carcinoma treated with definitive chemoradiotherapy. Sci Rep. 2017;7:42581.

7. Chen MF, Chen PT, Kuan FC, et al. The predictive value of pretreatment neutrophil-to-lymphocyte ratio in esophageal squamous cell carcinoma. Ann Surg Oncol. 2019;26:190-9.

8. Song Q, Wu JZ, Jiang HF, et al. The postoperative lymphocyte to monocyte ratio change predicts poor clinical outcome in patients with esophageal squamous cell carcinoma undergoing curative resection. Dis Markers. 2020;2020:1451864.

9. Barbetta A, Nobel TB, Sihag S, et al. Neutrophil to lymphocyte ratio as predictor of treatment response in esophageal squamous cell cancer. Ann Thorac Surg. 2018;106(3):864-71.

10. Sun Y, Zhang L. The clinical use of pretreatment NLR, PLR, and LMR in patients with esophageal squamous cell carcinoma: evidence from a meta-analysis. Cancer Manag Res. 2018;10:6167-79.

11. Zhang X, Jiang Y, Wang Y, et al. Prognostic role of neutrophillymphocyte ratio in esophageal cancer: a systematic review and meta-analysis. Medicine Baltimore. 2018;97(49):e13585.

12. Pirozzolo G, Gisbertz S, Castoro C, et al. Neutrophil-to-lymphocyte ratio as prognostic marker in esophageal cancer: a systematic review and meta-analysis. J Thorac Dis. 2019;11(7).

13. Proctor MJ, Morrison DS, Talwar D, et al. An inflammationbased prognostic score (mGPS) predicts cancer survival independent of tumour site: a Glasgow Inflammation Outcome Study. Br J Cancer. 2011;104:726-34.

14. Hoshino S, Takeuchi M, Kawakubo H, et al. Usefulness of neutrophil to lymphocyte ratio at recurrence for predicting longterm outcomes in patients with recurrent esophageal squamous cell carcinoma. Ann Surg Oncol. 2021. https://doi.org/10.1245/ s10434-021-09637-0.

15. Tanoglu A, Karagoz E, Yiyit N, et al. Is combination of neutrophil to lymphocyte ratio and platelet lymphocyte ratio a useful predictor of postoperative survival in patients with esophageal squamous cell carcinoma? Onco Targets Ther. 2014;7:433-4.

16. Azad TD, Chaudhuri AA, Fang P, et al. Circulating tumor DNA analysis for detection of minimal residual disease after chemoradiotherapy for localized esophageal cancer. Gastroenterology. 2020;158(3):494-505.e6.

17. Jia R, Zhao CH, Li PS, et al. Post-radiation circulating tumor DNA as a prognostic factor in locally advanced esophageal squamous cell carcinoma. Oncol Lett. 2021;21(1):68.

18. Ueda M, Iguchi T, Masuda T, et al. Somatic mutations in plasma cell-free DNA are diagnostic markers for esophageal squamous cell carcinoma recurrence. Oncotarget. 2016;20;7(38):62280-91.

Publisher's Note Springer Nature remains neutral with regard to jurisdictional claims in published maps and institutional affiliations. 